\title{
Cellular immune monitoring for prediction of cytomegalovirus and BK viral reactivation after kidney transplantation
}

\author{
Kyung-Hwa Shin ${ }^{1}$, Jin Hyeon Lee ${ }^{1}$, Sang Heon Song ${ }^{2}$, Eun Young Seong ${ }^{2}$, Miyeun Han², Hyung-Hoi Kim \\ ${ }^{1}$ Department of Laboratory Medicine, Pusan National University Hospital, Busan, Korea \\ ${ }^{2}$ Division of Nephrology, Department of Internal Medicine, Pusan National University Hospital, Busan, Korea
}

Background: The reactivation of cytomegalovirus (CMV) and BK virus after kidney transplantation is a critical factor that affects donor and graft survival. However, accurate methods for viral reactivation prediction and overall immune status assessment after transplantation remain unavailable. This study aimed to evaluate cellular immunity assays based on the potential for predicting $\mathrm{CMV}$ and $\mathrm{BK}$ viral reactivation in kidney transplant patients.

Methods: Fourteen male and twelve female patients who underwent kidney transplant were enrolled in the study between November 2019 and June 2020 (median age: 58 years [range, 38-68 years]). Whole blood samples were collected before transplantation. QuantiFERON-CMV (Qiagen, Germantown, MD, USA), QuantiFERON-Monitor assay (Qiagen), and lymphocyte subsets (CD3, CD4, CD8, CD19, CD56) were used to quantify the cellular immune response.

Results: There were no differences between the lymphocyte subsets from patients with and without CMV viremia. However, the levels of T lymphocytes in patients without BK viremia (median CD3+ 71.2\%) was higher than those in patients with BK viremia (median CD3+62.5\%). The interferon gamma (IFN- $\gamma$ ) levels (measured using the QuantiFERON-Monitor) in patients with CMV viremia (median, $160.9 \mathrm{IU} / \mathrm{mL}$ ) and in those without CMV viremia (median, $324 \mathrm{IU} / \mathrm{mL}$ ) did not differ. Also, the IFN- $\gamma$ levels in patients with BK viremia (median, $256.5 \mathrm{IU} / \mathrm{mL}$ ) and in those without BK viremia (median, $231.3 \mathrm{lU} / \mathrm{mL}$ ) did not differ. The patients with CMV viremia $(n=8)$ exhibited reactivity in the QuantiFERON-CMV assay. The patients with non-reactive QuantiFERON-CMV profile $(n=7)$ results did not show viremia.

Conclusions: Immune monitoring and the prediction of reactivation risk after kidney transplantation are critical factors in kidney transplant. The combination of these methods is useful for predicting CMV and BK viral reactivation after solid organ transplantation.

Corresponding author: Kyung-Hwa Shin

E-mail: skh2009pnuh@gmail.com

(C) The Korean Society for Transplantation

This is an Open Access article distributed under the terms of the Creative Commons Attribution Non-Commercial License (http://creativecommons.org/licenses/by-nc/4.0/) which permits unrestricted non-commercial use, distribution, and reproduction in any medium, provided the original work is properly cited. 